\title{
Shock Waves in Transonic Channel Flows at Moderate Reynolds Numbers
}

\author{
J. L. Mace* \\ Air Force Wright Aeronautical Laboratories, Wright-Patterson Air Force Base, Ohio \\ and \\ T. C. Adamson $\mathrm{Jr} . \dagger$ \\ University of Michigan, Ann Arbor, Michigan
}

\begin{abstract}
The behavior of shock waves in transonic channel flows with varying Reynolds and Prandtl numbers is examined using analytical and numerical methods. It is shown that the location of the sonic line within the structure of a shock wave is independent of the Reynolds number and is coincident with the location of the corresponding discontinuous wave in the limit as the Reynolds number tends to infinity. Also, in a numerical solution, truncation errors and artificial viscosity produce a smeared shock wave similar to that found in a flow at a moderate Reynolds number. Thus, these results lend support to the commonly accepted supposition that the position of the sonic line within the structure of a numerical shock wave can be adopted as the location of the corresponding shock wave in inviscid flow.
\end{abstract}

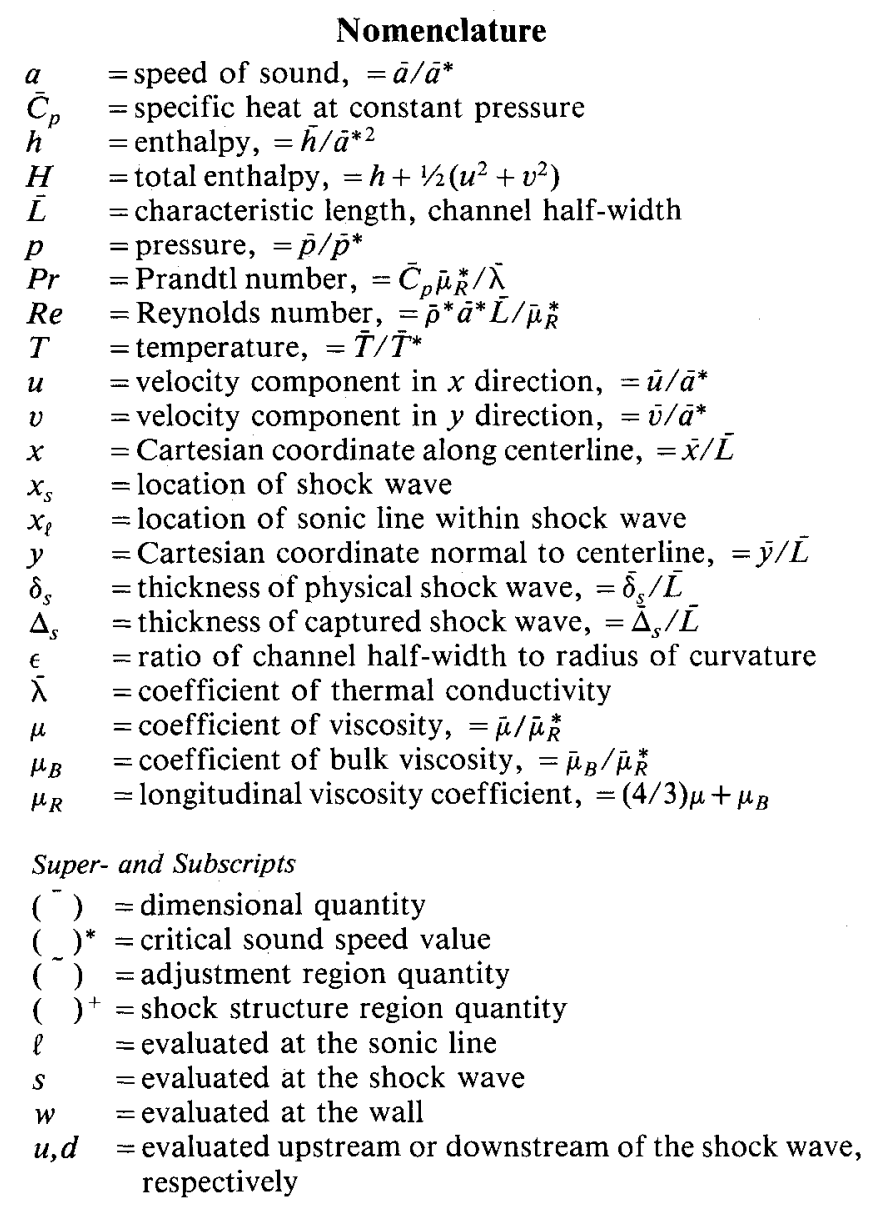

Received Nov. 29, 1984; presented as Paper 85-0369 at the AIAA 23rd Aerospace Sciences Meeting, Reno, NV, Jan. 14-17, 1985; revision received Aug. 13, 1985. This paper is declared a work of the U.S. Government and is not subject to copyright protection in the United States.

*Aerospace Engineer. Senior Member AIAA.

$\dagger$ Professor, Chairman, Department of Aerospace Engineering. Fellow AIAA.

\section{Introduction}

$\mathbf{T}$ HE numerical simulation of transonic flows involving shock waves is largely accomplished using shockcapturing finite difference or finite volume methods. Such methods are advantageous for simulating compressible flows since they allow a shock wave, whose location and strength are unknown in advance, to evolve as part of an initial value problem. However, these methods give rise to solutions exhibiting oscillations in the neighborhood of a captured shock wave and the thickness of the captured wave is significantly larger than that of the physical shock wave at a high Reynolds number. The ability to determine the location of a discontinuous shock wave within a numerical solution is important in obtaining acceptable aerodynamic simulations. For example, this information is crucial in determining the aerodynamic forces and moments on wings and cascade blades, especially in unsteady flows. Unfortunately, because of the finite thickness of a captured wave, it is difficult, at this time, to determine the location of the shock wave with the desired measure of simplicity and accuracy.

The smearing of a captured shock wave is the result of dissipative phenomena arising from both the truncation error in the numerical scheme and the diffusive terms (referred to as the "artificial viscosity") explicitly included in the difference equations. As a result of truncation errors and artificial viscosity, the actual differential equations simulated by the difference equations are no longer the original governing equations but, instead, are a set of modified equations. These modified equations contain higher-order spatial derivatives that introduce dissipative and dispersive phenomena into the solution. ${ }^{1} \mathrm{~A}$ common practice is to diminish the oscillatory behavior of the solution by increasing the magnitude of the artificial viscosity in the neighborhood of the shock wave, thereby producing a nearly monotonic transition of the flow properties through the shock structure. Evidently, under these conditions, the dissipative terms in the modified governing equations are large in magnitude compared to the dispersive terms. In general, the thickness of a captured shock wave may be characterized as occurring over 3-10 grid points of a numerical solution containing 30-100 streamwise grid points defining a characteristic body length $\bar{L}$. Hence, as an upper bound, a numerical estimate for the thickness $\Delta_{s}$ of a captured shock wave, normalized by $\bar{L}$, is 0.1 . 
In a flow described by a preshock Mach number $M_{u}$ and a Reynolds number $R e$ based on $\bar{L}$, a shock wave has thickness $^{2} \delta_{s}$ normalized by $L$, of $\mathcal{O}\left[\operatorname{Re}^{-1}\left(M_{u}-1\right)^{-1}\right]$; for typical transonic flows at high Reynolds number, the thickness of a shock is characterized by the mean free path of the gas molecules. Obviously, the thickness of a captured shock wave is much larger than that of a corresponding wave at high Reynolds number. That is, shock-capturing methods model the structure of a shock wave corresponding to a flow at a much lower Reynolds number, say

$$
\tilde{R} e=\mathcal{O}\left[\Delta_{s}^{-1}\left(M_{u}-1\right)^{-1}\right]
$$

Numerical solutions of shock waves in transonic flows exhibit thicknesses corresponding to moderate Reynolds numbers of $10^{2}-10^{3}$. Thus, $\tilde{R} e$ is small in comparison to the desired high Reynolds number, yet large in comparison with unity.

A shock wave at high Reynolds number, which appears as a discontinuity, should evolve as the Reynolds number tends toward infinity from a thick shock wave structure corresponding to a moderate Reynolds number. It is clear that the thickness varies inversely with Reynolds number, but there seems to be no known experimental or analytical evidence indicating the dependence upon Reynolds number of the position of the sonic line within the shock wave structure. Numerical results 3,4 have indicated that, for a practical range of computational parameters, the location of the sonic line within a captured shock wave does not vary with the magnitude of the artificial viscosity. It has been common practice (e.g., Ref. 3) to identify the position of the sonic line within the captured shock wave with that of the desired discontinuity. In light of these observations, several hypotheses are examined in this study: 1) to the accuracy desired, the location of the sonic line within the structure of a shock wave in transonic flow is independent of Reynolds number and so coincident with the location of the corresponding discontinuous wave in the limit as the Reynolds number tends to infinity and 2) in a numerical solution, truncation errors and added terms that introduce artificial viscosity produce a smeared shock wave similar to that found in a flow at moderate Reynolds numbers such that the location of the sonic line corresponds to the position of the desired shock wave at high Reynolds numbers.

The validation of these hypotheses is accomplished by comparing analytical and numerical solutions for a transonic flow in a symmetric channel. First, known solutions for inviscid flow are extended to include a relation for the position of the shock wave to first order. Second, analytical solutions for a viscous transonic flow in the channel are derived. These solutions include the location of the sonic line within the thick shock structure, which is compared with the location of the shock wave when the flow is inviscid. Finally, numerical solutions of an inviscid transonic flow in the channel are obtained and the location and structure of the captured shock wave are compared with the results found from the analytical solutions.

\section{Formulation of Problem}

The model problem chosen is that of a steady, transonic flow in a converging-diverging, symmetric channel as shown in Fig. 1. Far upstream and downstream of the channel throat, the walls are parallel and eventually connect to plenum chambers. The flow far upstream is uniform and subsonic; it accelerates through a sonic throat to supersonic speeds. The supersonic flow is terminated by a shock wave residing in the diverging portion of the channel. The subsonic flow downstream of the shock wave decelerates into that portion of the channel with parallel walls.

The coordinates $x$ and $y$ measured along and normal to the channel centerline, with $x=0$ at the point of minimal width, are made dimensionless by half the minimum channel

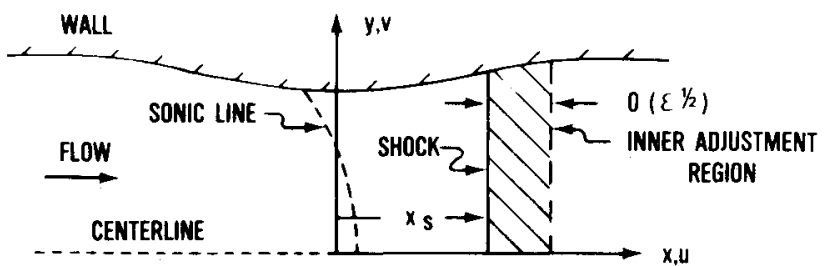

Fig. 1 Geometry and inviscid flow characteristics.

width $\bar{L}$. The effective shape of the channel walls, which may include the boundary-layer displacement thickness, is given by

$$
y_{w}(x)= \pm\left[1+\epsilon^{2} f(x)\right]
$$

where $f(0)=f^{\prime}(0)=0$ and $\epsilon \ll 1$. Here $\epsilon^{2}$ is defined as the ratio of the channel half-width to the product of the radius of curvature at the channel throat and $f^{\prime \prime}(0)$.

The gas is assumed to obey the perfect-gas law and have constant specific heats. The density $\rho$, pressure $p$, and temperature $T$ are made dimensionless with respect to their undisturbed critical values. The enthalpy $h$ and internal energy $\boldsymbol{e}$ are normalized by $\bar{a}^{* 2}$, while the entropy $s$ is made dimensionless with respect to the specific gas constant $\bar{R}$. The $x$ and $y$ velocity components $u$ and $v$, respectively, are made dimensionless by $\vec{a}^{*}$. Here an overbar denotes a dimensional quantity.

The equations governing the motion of the flow are the continuity, the Navier-Stokes, and the energy equations, written $^{5}$ as

$$
\begin{gathered}
(\rho u)_{x}+(\rho v)_{y}=0 \\
\rho u u_{x}+\rho v u_{y}=-\frac{1}{\gamma} p_{x}+\frac{1}{\operatorname{Re}} R(u) \\
\rho u v_{x}+\rho v v_{y}=-\frac{1}{\gamma} p_{y}+\frac{1}{R e} R(v) \\
\rho u H_{x}+\rho v H_{y}=\frac{1}{\operatorname{Re}}[R(h)+u R(u)+v R(v)]
\end{gathered}
$$

where $H$ is the total enthalpy and $\gamma$ the ratio of specific heats. The momentum transport terms $R(u)$ and $R(v)$ are defined as

$$
R(u)=\left[\mu_{R} u_{x}-\left(2 / 3 \mu-\mu_{B}\right) v_{y}\right]_{x}+\left[\mu\left(u_{y}+v_{x}\right)\right]_{y}
$$

and

$$
R(v)=\left[\mu\left(u_{y}+v_{x}\right)\right]_{x}+\left[\mu_{R} v_{y}-\left(2 / 3 \mu-\mu_{B}\right) u_{x}\right]_{y}
$$

and the thermal transport term $R(h)$ is

$$
\begin{aligned}
& R(h)=\left(\frac{\mu_{R}}{P_{r}} h_{x}\right)_{x}+\left(\frac{\mu_{R}}{P_{r}} h_{y}\right)_{y}+\mu_{R}\left(u_{x}^{2}+v_{y}^{2}\right) \\
& -\left(\frac{4}{3} \mu-2 \mu_{B}\right) u_{x} v_{y}+\mu\left(u_{y}+v_{x}\right)^{2}
\end{aligned}
$$

where the coefficient of viscosity $\mu$ and the bulk viscosity coefficient $\mu_{B}$ are used to define the longitudinal viscosity coefficient $\mu_{R}$.

The equation of state, in dimensionless form, is

$$
p=\rho T
$$


Finally, the above equations can be combined to form the gasdynamic equation

$$
\begin{aligned}
& \left(u^{2}-a^{2}\right) u_{x}+u v\left(u_{y}+v_{x}\right)+\left(v^{2}-a^{2}\right) v_{y} \\
& =\frac{1}{\rho R e}[u R(u)+v R(v)-(\gamma-1) R(h)]
\end{aligned}
$$

which will be used extensively in the following sections

To complete the problem formulation, boundary conditions must be specified for the flow far upstream and downstream of the channel throat as well as along the channel walls. Since it is only the details of the flowfield external to the boundary layer that are desired, the boundary condition for $v$ along the walls is written as

$$
v\left(x, y_{w}\right)= \pm \frac{\mathrm{d} y_{w}}{\mathrm{~d} x} u\left(x, y_{w}\right)
$$

where $y_{w}$ is assumed to include the displacement thickness of the boundary layer; i.e., the physical wall shape is such that, after the displacement thickness has been accounted for, the resulting effective shape is given by Eq. (1). As will be seen, terms containing the viscosity, i.e., the Reynolds number, occur as known forcing functions at each order of approximation so that no boundary condition on $u$ at the wall is needed to the order retained. The pressure in the channel far downstream of the shock wave is adjusted such that a shock wave resides in the diverging portion of the channel. The pressure in the channel far upstream of the throat is determined from the condition that the flow is sonic at the throat.

\section{Inviscid, Transonic Flow with Shock Wave}

Analytical solutions for a steady, inviscid, transonic flow containing a shock wave in a two-dimensional channel have been obtained by Messiter and Adamson. ${ }^{6}$ These solutions are in the form of asymptotic expansions valid to second order; the location of the shock wave is obtained to zeroth order. Here, the solutions for the flow velocities are extended to third order, while the location of the discontinuous shock wave, $x_{s}(y)=\mathcal{O}(1)$, is found to first order.

The governing equations for this problem are those obtained from Eqs. (2-5) in the limit as the Reynolds number tends to infinity. With this assumption, the gasdynamic equation (10) becomes

$$
\left(u^{2}-a^{2}\right) u_{x}+u v\left(u_{y}+v_{x}\right)+\left(v^{2}-a^{2}\right) v_{y}=0
$$

where the sound speed $a$ is obtained from the energy equation

$$
\frac{a^{2}}{\gamma-1}+\frac{u^{2}+v^{2}}{2}=\frac{\gamma+1}{2(\gamma-1)}
$$

For a shock wave in transonic flow, the velocity components upstream and downstream of the wave are related by the transonic approximation ${ }^{6}$ to the shock polar equation given by

$$
\left(v_{d}-v_{u}\right)^{2} \simeq 1 / 2(\gamma+1)\left(u_{u}-u_{d}\right)^{2}\left(u_{u} u_{d}-1\right)
$$

where the subscripts $u$ and $d$ refer to values immediately upstream and downstream of the shock wave, respectively.

Composite asymptotic expansions for the velocity components may be written ${ }^{7}$ as

$$
\begin{array}{r}
u(x, y ; \epsilon) \sim 1+\epsilon u_{1}(x)+\epsilon^{2}\left[u_{2}(x, y)+\xi_{\tilde{x}}(\tilde{x}, \tilde{y})\right] \\
+\epsilon^{5 / 2} \beta_{\tilde{x}}(\tilde{x}, \tilde{y})+\epsilon^{3}\left[u_{3}(x, y)+\eta_{\tilde{x}}(\tilde{x}, \tilde{y})\right]+\ldots
\end{array}
$$

and

$$
\begin{gathered}
v(x, y ; \epsilon) \sim \epsilon^{2} v_{2}(x, y)+\epsilon^{5 / 2} \xi_{\tilde{y}}(\tilde{x}, \tilde{y}) \\
+\epsilon^{3}\left[v_{3}(x, y)+\beta_{\tilde{y}}(\tilde{x}, \tilde{y})\right]+\ldots
\end{gathered}
$$

where $\xi, \beta$, and $\eta$ are potential functions representing perturbation potentials for an adjustment region with length $\mathcal{O}\left(\epsilon^{1 / 2}\right)$ immediately downstream of the shock wave. Upstream of the shock wave, these potentials are identically zero. Suitable coordinates for the adjustment region are found to be $\tilde{x}=\left(x-x_{s}\right) / \epsilon^{1 / 2}$ and $\tilde{y}=y$. Asymptotic expansions valid for the temperature, density, and pressure are similar in form to that for the $u$ velocity component. The expansion for the position of the shock wave is found to be ${ }^{6}$

$$
x_{s}(y) \sim x_{s 0}+\epsilon x_{s 1}+\epsilon^{3 / 2} x_{s 3 / 2}(y)+\ldots
$$

indicative of a normal shock wave to $\Theta(\epsilon)$. Using these expansions, one can show the flow is irrotational to $\mathcal{O}\left(\epsilon^{3}\right)$, i.e., $v_{x}-u_{y}=\mathcal{O}\left(\epsilon^{4}\right)$. Substitution of these expansions into the governing equations (12-14) results in a sequence of differential equations that, together with the boundary conditions, are solved to give the results

$$
\begin{gathered}
u_{1}(x)= \pm\left(\frac{2}{\gamma+1} f(x)+c_{w}\right)^{1 / 2} \\
u_{2}(x, y)=\frac{3-2 \gamma}{6} u_{1}^{2}+f^{\prime \prime}(x)\left(\frac{y^{2}}{2}-\frac{1}{6}\right)+\frac{c_{2}}{u_{1}}
\end{gathered}
$$

and

$$
\begin{aligned}
& u_{3}(x, y)=\frac{\gamma+1}{6}\left(u_{1}^{3}\right)^{\prime \prime}\left(y^{2}-\frac{1}{3}\right) \\
& +\frac{\gamma+1}{6}\left(u_{1} f^{\prime \prime}\right)^{\prime \prime}\left(\frac{y^{4}}{4}-\frac{y^{2}}{2}+\frac{7}{60}\right) \\
& +\frac{\gamma^{2}-12 \gamma+9}{36} u_{1}^{3}+\frac{3-2 \gamma}{3} c_{2}-\frac{1}{90} \frac{f^{\prime \prime} 2}{u_{1}} \\
& -\frac{c_{2}^{2}}{2 u_{1}^{3}}-\frac{f^{\prime 2}}{6(\gamma+1) u_{1}}-\frac{f^{2}}{2(\gamma+1) u_{1}}+\frac{c_{3}}{u_{1}}
\end{aligned}
$$

for $u_{1}, u_{2}$, and $u_{3}$, respectively. Also, the transverse velocity components are

$$
v_{2}(x, y)=f^{\prime}(x) y
$$

$$
v_{3}(x, y)=(\gamma+1) u_{1}^{2} u_{1 x} y+(\gamma+1)\left(u_{1} f^{\prime \prime}\right)^{\prime}\left(y^{2}-1\right)(y / 6)
$$

The constant $c_{w}$ in Eq. (18) is set by the velocity at the channel throat: since the throat is choked for the problem being considered, then $u_{1}^{2}(0)=c_{w}=0$. The constants $c_{2}$ and $c_{3}$ in Eqs. (19) and (20), respectively, are set by specifying the channel inflow and outflow conditions. It should be noted that for the present case, where $u_{1}(0)=0, c_{2 u}=0$ and $c_{3 u}=f^{\prime \prime 2}(0) / 90$. The upper and lower signs in Eq. (18) correspond to locally supersonic and subsonic flow conditions, respectively. The perturbation potential $\xi(\tilde{x}, \tilde{y})$ is given by

$$
\begin{aligned}
& \xi(\tilde{x}, \tilde{y})=4 f_{0}^{\prime \prime} \sqrt{(\gamma+1) u_{10}} \sum_{n=1}^{\infty}\left\{\frac{(-1)^{n}}{(n \pi)^{3}}\right. \\
& \left.\quad \times \cos n \pi \tilde{y} \exp \left[-n \pi \tilde{x} / \sqrt{(\gamma+1) u_{10}}\right]\right\}
\end{aligned}
$$

which is that given by Messiter and Adamson. ${ }^{6}$ The notation $u_{10}$ is defined here as $u_{1}\left(x_{s 0}\right)$.

The location of the shock wave can be determined by equating the mass flow rates at locations upstream and 
downstream of the shock wave in a manner outlined by Messiter and Adamson. ${ }^{6} \mathrm{~A}$ control volume bounded by surfaces at the channel throat, the channel wall and centerline and convenient location far downstream of the shock wave, where the flow is uniform, is considered; then, the requirement that mass be conserved gives an integral relation relating flow properties upstream and downstream of the shock wave. The thermodynamic property equation and the energy equation may be used to relate $\rho$ and $u$ in this integral relation, while the Rankine-Hugoniot relations are used to determine the entropy increase across the shock wave. ${ }^{6}$ By expanding each term in the resulting equation in a Taylor series about $x_{s} \sim x_{s 0}+\epsilon x_{s 1}+\ldots$ and integrating, one finds, as $\epsilon \rightarrow 0$, the results

$$
c_{2 d}=c_{2 u}-\frac{2 \gamma}{3} u_{10}^{3}
$$

and

$$
c_{3 d}=c_{3 u}+\frac{2 \gamma^{2}}{3} u_{10}^{4}-2 \gamma x_{s 1} u_{10}^{2} u_{1 x 0}-2 \gamma u_{10} c_{2 u}
$$

The position of the shock wave to lowest order $x_{50}$ may be determined from Eq. (24). The specification, for example, of the pressure to second order at the downstream flow boundary is equivalent to setting $c_{2 d}$. Hence, Eq. (24) can be used to calculate $u_{1}\left(x_{s 0}\right)$ from which $x_{50}$ can be determined using Eq. (18). In a similar manner, $x_{s 1}$ can be calculated using Eq. (25). The specification of the pressure to third order at the downstream flow boundary determines the constant $c_{3 d}$. Hence, the first-order shock wave position $x_{s 1}$ can be calculated explicitly from Eq. (25).

The results presented here are seen to be extensions of the solutions obtained by Messiter and Adamson ${ }^{6}$ to higherorder terms. Specifically, the outer velocity solutions have been extended to include the third-order terms, $u_{3}$ and $v_{3}$ given by Eqs. (20) and (22), respectively. Also, the shock wave location $x_{s}$ has been extended to include the first-order term $x_{s 1}$ given by Eq. (25). Finally, the solutions presented here, i.e., Eqs. (15) and (16), may be used to validate numerical methods since the solutions describe a demanding nonlinear transonic flow.

\section{Viscous Transonic Flow with Shock Wave}

The structure of a weak normal shock wave was described by Taylor $^{8}$ for a perfect gas with constant transport coefficients. Higher-order approximations, in which the Taylor solution appeared as the first approximation, were presented by Szaniawski. ${ }^{9}$ The structure of two-dimensional shock waves was described by $\operatorname{Sichel}^{10}$ as a solution to the viscous transonic equation. Similarity solutions for shock waves in a transonic channel flow at moderate Reynolds number were also presented by Sichel. ${ }^{11}$ These solutions, while clearly showing the influence of Reynolds number on the structure, lacked generality in that only special wall shapes could be considered. These restrictions were removed by Adamson and Messiter ${ }^{12}$ who obtained solutions, in the form of asymptotic expansions, for a shock wave in a channel flow at moderate Reynolds number and constant Prandtl number. The work presented here extends that of Adamson and Messiter $^{12}$ to include third-order solutions with variable Prandtl number, which are then used to find the location of the sonic line within the viscous shock wave to first order.

The steady, transonic flow of a viscous and thermally conducting perfect gas in a symmetric channel (Fig. 2) is described by Eq. (10). For simplicity, the Stokes relation between the first and the second coefficients of viscosity is assumed thus implying that the bulk viscosity is zero. ${ }^{13}$ The viscosity coefficient $\mu$ is assumed to be described ${ }^{14}$ by the power law relation $\mu=T^{k_{v}}$, where $k_{v}=\Theta(1)$. The problem to be examined is one in which the Reynolds number is taken as $R e^{-1}=k_{s} \epsilon^{2}$ where $k_{s}=\mathcal{O}(1)$. With this Reynolds number scaling, it will be found that the flow to lowest order is inviscid.

Outer composite expansions for the velocity components are given $\mathrm{by}^{7}$

$$
\begin{gathered}
u\left(x, y ; \epsilon, k_{s}\right) \sim 1+\epsilon u_{1}(x)+\epsilon^{2}\left[u_{2}\left(x, y ; k_{s}\right)+\xi_{\tilde{x}}\right] \\
+\epsilon^{5 / 2} \beta_{\bar{x}}+\epsilon^{3}\left[u_{3}\left(x, y ; k_{s}\right)+\eta_{\bar{x}}\right]+\ldots
\end{gathered}
$$

and

$$
v\left(x, y ; \epsilon, k_{s}\right) \sim \epsilon^{2} v_{2}(x, y)+\epsilon^{5 / 2} \xi_{\bar{y}}+\epsilon^{3}\left[v_{3}(x, y)+\beta_{\tilde{y}}\right]+\ldots
$$

where $\xi, \beta$, and $\eta$ are perturbation potentials arising in an adjustment region of extent $\tilde{x}=\left(x-x_{s}\right) / \epsilon^{1 / 2}=\mathcal{O}(1)$ and $\tilde{y}=y=\mathcal{O}(1)$ occurring immediately downstream of the shock structure region; upstream of the structure region the perturbation potentials are zero, as found by Messiter and Adamson. ${ }^{6}$ The thermodynamic variables have expansions similar to the $u$ velocity component.

In the outer composite regions, it is found that $R(u)=\mathcal{O}(\epsilon), \quad R(v)=\mathcal{O}\left(\epsilon^{2}\right)$, and $R(h)=\mathcal{O}(\epsilon)$ so that $H=1 / 2(\gamma+1) /(\gamma-1)+\mathcal{O}\left(\epsilon^{3}\right)$, but the term $\mathcal{O}\left(\epsilon^{3}\right)$ and its counterpart in the expansion for the entropy are both functions of $x$ alone, so the flow is irrotational to $\mathcal{O}\left(\epsilon^{4}\right)$. Substitution of these relations into the gasdynamic equation (10) and the vorticity relation $v_{x}-u_{y}=\mathcal{O}\left(\epsilon^{4}\right)$ results in six differential equations that, when combined with the wall boundar $y$ conditions, allow the determination of the velocity components. The velocity components $u_{1}, v_{2}$, and $v_{3}$ remain unchanged from those found for the inviscid flow, Eqs. (18), (21), and (22), respectively. Additionally, the perturbation potential $\xi$ remains unchanged and is given by Eq. (23). It is the higher-order velocity components $u_{2}$ and $u_{3}$ that are modified by viscosity; these components are given by

$$
u_{2}(x, y)=f^{\prime \prime}(x)\left(\frac{y^{2}}{2}-\frac{1}{6}\right)+\frac{3-2 \gamma}{6} u_{1}^{2}+\frac{c_{2}+m u_{1 x}}{u_{1}}
$$

and

$$
\begin{aligned}
& u_{3}(x, y)=(\gamma+1)\left(\frac{u_{1}^{3}}{3}\right)^{\prime \prime} \frac{y^{2}}{2}+(\gamma+1)\left(u_{1} f^{\prime \prime}\right)^{\prime \prime}\left(\frac{y^{4}}{24}-\frac{y^{2}}{12}\right) \\
& +(\gamma+1)\left[\frac{7 u_{1} f^{\prime \prime}}{360}-\frac{u_{1}^{3}}{18}\right]^{\prime \prime}+\frac{\gamma^{2}-12 \gamma+9}{36} u_{1}^{3} \\
& +\frac{3-2 \gamma}{3} c_{2}-\frac{1}{u_{1}}\left[\frac{f^{2}}{2(\gamma+1)}+\frac{f^{\prime 2}}{6(\gamma+1)}+\frac{f^{\prime \prime}}{90}-c_{3}\right] \\
& -\frac{\left(m u_{1 x}+c_{2}\right)^{2}}{2 u_{1}^{3}}-m\left\{k_{v}(\gamma-1) u_{1 x}+\frac{B(\gamma-1)(P r-1)}{\operatorname{Pr}+\gamma-1}\right. \\
& +\frac{1}{u_{1}}\left[\left(\gamma-\frac{(\gamma-1)(P r-1)}{\operatorname{Pr}+\gamma-1}\right) \int_{x_{I}}^{x} u_{1 x}^{2} d x\right. \\
& \left.\left.\left.-\left(f+\frac{6-\gamma}{6} u_{1}^{2}+\frac{c_{2}+m u_{1 x}}{u_{1}}\right)\right]_{x}\right]\right\}
\end{aligned}
$$

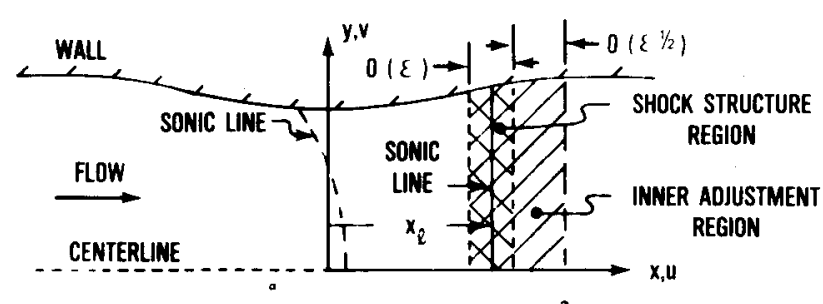

Fig. 2 Viscous flow regions for $\epsilon^{2} R e=\mathcal{O}(1)$. 
respectively. Here $m$ is defined as

$$
m=\frac{4 k_{s}}{3(\gamma+1)}\left(1+\frac{\gamma-1}{\operatorname{Pr}}\right)
$$

and is $O(1)$. The constant $B$ arises from the evaluation of the total enthalpy and is defined by $B=u_{1 x}(0)$. The lower limit of integration $x_{1}$ found in Eq. (29) must be consistent with the integration constants $c_{2}$ and $c_{3}$.

Just as was found in the inviscid flow, an adjustment region of $\mathcal{O}\left(\epsilon^{1 / 2}\right)$ in extent downstream of the shock structure is required in order to match successfully the outer solutions with the structure solutions. If one defines a perturbation potential, $\phi(\tilde{x}, \tilde{y})$, so that

$$
\Phi(\tilde{x}, \tilde{y})=\epsilon^{5 / 2} \xi(\tilde{x}, \tilde{y})+\epsilon^{3} \beta(\tilde{x}, \tilde{y})+\epsilon^{7 / 2} \eta(\tilde{x}, \tilde{y})+\ldots
$$

then the gasdynamic equation becomes

$$
\Phi_{\tilde{r} \tilde{r}}+\Phi_{\tilde{y} \tilde{y}}+Q_{\tilde{r}}=0, \quad \tilde{r}>0
$$

where $\tilde{r}=\tilde{x} /\left[(\gamma+1) u_{10}\right]^{1 / 2}, \tilde{y}=y$, and $Q_{r}$ is composed of known lower-order velocity components at each order of approximation. ${ }^{7}$ The wall and centerline boundary conditions become $\Phi_{\bar{y}}(\tilde{x}, 1)=\Phi_{\bar{y}}(\tilde{x}, 0)=0$, respectively, while matching to the outer downstream solutions requires

$$
\Phi_{\bar{r}} \rightarrow 0 \text { as } \tilde{r} \rightarrow \infty
$$

Application of the divergence theorem ${ }^{15}$ to Eq. (32) gives, as $\epsilon \rightarrow 0$, the relations

$$
\begin{aligned}
& \int_{0}^{1} \xi_{\tilde{x}}(0, \tilde{y}) \mathrm{d} \tilde{y}=0 \\
& \int_{0}^{1} \beta_{\tilde{x}}(0, \tilde{y}) \mathrm{d} \tilde{y}=0
\end{aligned}
$$

and

$$
\int_{0}^{1} \eta_{\tilde{x}}(0, \tilde{y}) \mathrm{d} \tilde{y}=\frac{-m}{u_{10}} \int_{0}^{1} \beta_{\tilde{x} \tilde{x}}(0, \tilde{y}) \mathrm{d} \tilde{y}+\frac{2}{\gamma+1} \int_{0}^{1} x_{\hat{\beta} / 2}^{\prime 2} \mathrm{~d} \tilde{y}
$$

which can be used to complete the specification of the boundary value problem for $\Phi$.

Since the problem chosen is that of a transonic flow in a dissipative gas with a Reynolds number $\mathcal{O}\left(\epsilon^{-2}\right)$, it is expected that a shock wave will appear as a region, with thickness $\mathcal{O}(\epsilon)$, in which the flow undergoes a rapid transition. The gas speed downstream of the shock wave is taken as subsonic so that a sonic line, located at $x_{p}(y)$, is within the shock wave structure. The sonic line certainly remains within the structure of the shock wave with increasing Reynolds number, thus permitting $x_{p}(y)$ to represent the location of the shock structure region. In the limit as Reynolds number goes to infinity, $x_{p}(y)$ must be coincident with the location of the discontinuous, inviscid flow shock wave; i.e., $x_{\ell}(y) \rightarrow x_{s}(y)$ as $R e \rightarrow \infty$. Evidently then, a suitable expansion for $x_{\ell}$ is

$$
x_{p}(y) \sim x_{p 0}+\epsilon x_{p 1}+\epsilon^{3 / 2} x_{p 3 / 2}(y)+\ldots
$$

The characteristic lengths associated with the shock structure region are the shock wave thickness, $\delta_{s}=\mathcal{O}(\epsilon)$, in the streamwise direction and the channel width, $y_{w}\left(x_{\ell}\right)=\mathcal{O}(1)$, in the transverse direction. Hence, independent variables, $x^{+}$and $y^{+}$, suitable for this inner region are taken as

$$
x^{+}=\frac{x-x_{\ell}(y)}{\epsilon}, \quad y^{+}=y
$$

where $x_{p}(y)$ is given by Eq. (36).
The forms of expansions for the velocity components in the inner structure region are found by expanding Eqs. (26) and (27) for the outer velocity components in Taylor series about the sonic line $x_{q}(y)$. It is found that proper expansions for the velocity components in the inner shock structure region are given by ${ }^{7}$

$$
\begin{gathered}
u \sim 1+\epsilon u_{1}^{+}+\epsilon^{2} u_{2}^{+}+\epsilon^{5 / 2} u_{5 / 2}^{+}+\epsilon^{3} u_{3}^{+}+\ldots \\
v \sim \epsilon^{2} v_{2}^{+}+\epsilon^{5 / 2} v_{5 / 2}^{+}+\epsilon^{3} v_{3}^{+}+\ldots
\end{gathered}
$$

The pressure, density, and temperature expansions for this inner region are similar in form to Eq. (38).

The transport terms appropriate for the structure region are found by substituting the above expansions into Eqs. (6-8) to give $R(u) \sim \mathcal{O}\left(\epsilon^{-1}\right), \quad R(v) \sim \mathcal{O}(1)$, and $R(h)$ $\sim \mathcal{O}\left(\epsilon^{-1}\right)$. The expansion for the total enthalpy in the structure region is given by $H \sim 1 / 2(\gamma+1) /(\gamma-1)+\mathcal{O}\left(\epsilon^{2}\right)$, while the entropy variation within the structure region is $S \sim \vartheta\left(\epsilon^{2}\right)$. Finally, one finds the variation of vorticity in the region is $\mathcal{O}\left(\epsilon^{5 / 2}\right)$. The governing equations for the velocity components are found from the gasdynamic equation (10) and the vorticity relation $v_{x}-u_{y} \sim \mathcal{O}\left(\epsilon^{5 / 2}\right)$. Thus, one finds that $u_{1}^{+}$is governed by

$$
u_{1}^{+} u_{1 x^{+}}^{+}=m u_{1 x^{+} x^{+}}^{+}
$$

which has a solution $u_{1}^{+}=g_{1}(y) \tanh r^{+}$where $r^{+}=f_{2}(y)$ $-1 / 2 g_{1}(y) x^{+} / m$. Using this solution in the vorticity relation, one finds by matching to the outer flow that $v_{2}^{+}\left(y^{+}\right)=f_{0}^{\prime} y^{+}$ and $g_{1}$ and $f_{2}$ are constants, say $c_{1}^{+}$and $c_{2}^{+}$, respectively. Finally, matching the inner solution for $u_{1}^{+}$to the outer composite solutions, both upstream $\left(x^{+} \rightarrow-\infty\right)$ and downstream $\left(x^{+} \rightarrow+\infty\right)$, leads to the result that

where

$$
u_{1}^{+}\left(x^{+}\right)=u_{10} \tanh r^{+}
$$

$$
r^{+}=c_{2}^{+}-\frac{u_{10}}{2 m} x^{+}
$$

and $u_{10}=u_{1}\left(x_{p 0}\right)$.

Here $c_{2}^{+}$, which determines the origin of the $r^{+}$coordinate, may be found from higher-order relations that locate the sonic line position to $\mathcal{O}(\epsilon)$. The solution for $u_{1}^{+}$given by Eq. (41) is Taylor's solution for the structure of a weak shock wave.

The governing equation for $u_{2}^{+}$is written in terms of $r^{+}$ and subsequently integrated to give ${ }^{7}$

$$
\begin{aligned}
u_{2}^{+} & =\frac{-4 m f_{0}^{\prime}}{(\gamma+1) u_{10}}\left[\frac{r^{+2}}{4 \cosh ^{2} r^{+}}+\frac{\mathrm{r}^{+} \tanh r^{+}}{2}-\frac{1+\tanh ^{2} r^{+}}{8}\right] \\
& +u_{10}^{2}\left[\frac{\tanh ^{2} r^{+}}{2}-\frac{\ln \cosh r^{+}}{\cosh ^{2} r^{+}}\right]+A_{1}^{+} \frac{\ln \cosh r^{+}}{\cosh r^{+}} \\
& +f_{1}^{+}(y)\left[\frac{r^{+}}{2 \cosh ^{2} r^{+}}+\frac{\tanh r^{+}}{2}\right]+\frac{f_{2}^{+}(y)}{\cosh ^{2} r^{+}}
\end{aligned}
$$

where $f_{1}^{+}$and $f_{2}^{+}$are functions of integration and

$$
A_{1}^{+}=u_{10}^{2}\left[k_{v}(\gamma-1)+\frac{\gamma-1}{\gamma+1} \frac{P r-1}{2 m^{2}}\left(\frac{4 k_{s}}{3 P r}\right)^{2}\right]
$$

Matching this result to the outer solutions allows one to determine $f_{1}^{+}$, while $f_{2}^{+}$must be determined by evaluating $u_{2}^{+}$ at $r^{+}=0$. By matching both upstream and downstream second-order terms of $u$ one finds two equations for $f_{1}^{+}(y)$, which are combined with Eq. (33) to yield

$$
c_{2 d}=c_{2 u}-\frac{2 \gamma}{3} u_{10}^{3}
$$


Equation (44) provides a relationship between the constants specified by the upstream and downstream boundary conditions $c_{2 u}$ and $c_{2 d}$, respectively, and the sonic line location $x_{20}$ to $\mathcal{O}(1)$. This equation for $x_{p 0}$ is seen to be identical to Eq. (24) for determining the location of the discontinuous shock wave $x_{s 0}$ to $\mathcal{O}(1)$.

Integration of the governing equation for $u_{3}^{+}$gives $^{7}$

$$
\begin{aligned}
& u_{3 r^{+}}^{+}+\frac{2}{u_{10}} u_{1}^{+} u_{3}^{+}=\frac{3}{u_{10}} u_{1}^{+2} u_{2}^{+}-\frac{u_{2}^{+2}}{u_{10}} \\
& -C_{1}^{+} u_{1}^{+}-C_{2}^{+} \int r^{+} u_{1}^{+} \mathrm{d} r^{+}+C_{3}^{+} u_{10} \ln \cosh r^{+} \\
& -C_{4} r^{+}-\mu_{1}^{+} u_{2 r^{+}}^{+}-\mu_{2}^{+} u_{1 r^{+}}^{+}+C_{5}^{+} \frac{u_{1}^{+4}}{4}+C_{6}^{+} \frac{u_{1 r}^{+2}}{2} \\
& +C_{7}^{+}\left(H_{3 r}^{+}-\mu_{1}^{+} u_{1}^{+} u_{1 r^{+}}^{+}+\mu_{1}^{+} H_{2 r}^{+}\right)+g_{8}^{+}(y)
\end{aligned}
$$

where $g_{8}^{+}$is a function of integration, and where $C_{1}^{+}, C_{2}^{+}$, $C_{3}^{+}, C_{4}^{+}, C_{5}^{+}, C_{6}^{+}$, and $C_{7}^{+}$are given in Ref. 7. The asymptotic forms of $u_{1}^{+}$and $u_{2}^{+}$for $r^{+} \rightarrow \pm \infty$, in conjunction with the asymptotic forms of $u_{3}^{+}$in the same limits as given by the outer composite solutions, can be used in Eq. (45) to obtain two equations, one in each limit, of the form

$$
\left(u_{3}^{+}\right)_{u, d}=A_{u, d} x^{+2}+B_{u, d} x^{+}+C_{u, d}
$$

Hence, if coefficients of like powers of $x^{+}$are equated in each equation, six equations are obtained, three for each limit.

It is found ${ }^{7}$ that the coefficients of $x^{+2}$ give equations that are identities and the coefficients of $x^{+}$result in equations for determining $x_{p 2}^{\prime}$. Finally, it is seen from Eq. (45) that at each limit, $r^{+} \rightarrow+\infty$ or $r^{+} \rightarrow-\infty$, the coefficients of $x^{+0}$ include $g_{8}(y)$, the function of the integration. If one of these equations is subtracted from the other, the resulting equation, containing both the upstream and downstream asymptotic forms of the solution for the flow through the thick shock wave, embodies the jump conditions applicable to the third-order terms in the velocity components. The desired expression for $x_{p 1}$ is found by integration of the resultant equation over the interval $0 \leq y \leq 1$, where integrals of the potential functions $\xi, \beta$, and $\eta$ are found using Eqs. (33-35). The result is

$$
\begin{aligned}
c_{3 d} & =c_{3 u}-2 \gamma u_{10} c_{2 u}+2 / 3 \gamma^{2} u_{10}^{4} \\
& -2 \gamma\left(x_{\ell 1}+\frac{2 m c_{2}^{+}}{u_{10}}\right) u_{10}^{2} u_{1 x 0}+\frac{4 m^{2} f_{0}^{\prime \prime} c_{2}^{+}}{(\gamma+1) u_{10}^{2}}
\end{aligned}
$$

Since $r^{+}=-u_{10}\left[x-x_{p 0}-\epsilon\left(x_{\ell 1}+2 m c_{2}^{+} / u_{10}\right)+\ldots\right] / 2 \epsilon m$, it is seen from this result that the only location in the shock structure which is independent of Reynolds number to $\mathcal{O}(\epsilon)$ occurs at the point where $c_{2}^{+}=0$, i.e., when $x=x_{p}+\mathcal{O}\left(\epsilon^{3 / 2}\right)$. Moreover, with $c_{2}^{+}=0$, it is seen that the equation here for $x_{\ell 1}$ is identical to that found for the position of the inviscid shock wave term $x_{s 1}$ in Eq. (25). Hence, it has been found that to $\mathcal{\theta}(\epsilon)$ the location of the sonic line within the structure of a transonic shock wave at moderate Reynolds number is independent of Reynolds number and is coincident with the location of the corresponding discontinuous wave found at high Reynolds number.

\section{Numerical Solutions of Inviscid Channel Flow}

In the previous sections, analytical solutions describing the behavior of both inviscid and viscous transonic flows in channels have been presented. It was shown that to $\mathcal{O}(\epsilon)$ the position of the sonic line within the shock wave structure coincides with the desired discontinuous shock wave in high
Reynolds number flow. In this section, numerical simulations of inviscid transonic flows within the channel are presented. The structure of the captured shock wave is compared with the analytical structure solutions and the location of the sonic line within the captured wave is also compared with that from the analytical solutions.

The grid system used in the computation is composed of a set of adjoining quadrilateral mesh cells that are of nearly uniform spacing except for those cells near the channel wall. The grid lines are body fitted and nearly orthogonal in all regions of the solution domain. A numerical procedure, due to Visbal and Knight, ${ }^{16}$ is used to generate the grid. In the examples considered, the axial dimensionless cell length is roughly 0.05 and the dimensionless transverse width varies from a minimum of 0.02 near the wall to a maximum of roughly 0.05 near the centerline. The mesh consists of 130 streamline cells and 24 transverse cells. The cross-sectional flow areas associated with the entrance and exit of the channel are the same; the area ratio between either of these locations and the throat is 1.12 , which corresponds to selecting $\epsilon=0.2$.

The explicit finite volume algorithm due to MacCormack and Paullay ${ }^{17}$ is used to integrate the Euler equations in time from an initial state until a steady-state solution is obtained. The algorithm has been used extensively to obtain solutions to compressible flow problems. The scheme is a second-order accurate, shock-capturing method, in which the shock waves are smeared. The dependent variables are integrated in time by repeatedly applying the finite volume operator to the dependent variables. The operator is a sequence of onedimensional, time-split finite-volume operators.

The inclusion of artificial viscosity in the finite volume equations is of particular interest to this study. Artificial viscosity is added in the form of a fourth-order pressure damping term due to MacCormack. ${ }^{18}$ This particular form of damping is widely used in shock-capturing numerical schemes to ensure stability and diminish oscillations of the solution in the neighborhood of shock waves and serves as a suitable representative of the methods that implement artificial viscosity. The term added to the finite volume equations is of the form

$$
\Delta t \Delta x^{3} \frac{\partial}{\partial x}\left[\alpha \frac{(|u|+a)}{4 p}\left|\frac{\partial^{2} p}{\partial x^{2}}\right| \frac{\partial U}{\partial x}\right]
$$

where $\alpha$ is a constant used to vary the magnitude of this term.

The initial conditions selected correspond to a uniform axial flow throughout the solution domain. The wall boundary condition is the flow tangency condition given by Eq. (11). On the channel centerline, the transverse velocity component $v$ is set equal to zero. The values of the pressure at the wall and centerline are obtained from the equation ${ }^{19}$

$$
\frac{\partial p}{\partial n}=-K \rho\left(u^{2}+v^{2}\right)
$$

where $n$ is the outward normal distance from the wall and $K$ the curvature of the wall or centerline. The stagnation pressure and temperature are specified at the upstream inflow boundary, while static pressure is prescribed at the downstream outflow boundary. One-dimensional, unsteady characteristic equations ${ }^{20}$ are used to describe the inflow and outflow wave phenomena. For all cases to be presented here, the downstream static pressure is prescribed, according to quasi-one-dimensional flow theory, at such a value so as to place a normal shock wave at the location $x=0.8$. The numerical results are obtained using an integration time step, which is 0.7 times the maximum-allowable time step given by the linearized stability analysis. Integration is carried out for a time period of roughly 20 times the characteristic time 
associated with convecting, at the critical sound speed, the gas across the solution domain (a dimensionless distance of approximately 6.5 )

First, the numerical simulation is compared with the viscous transonic flow theory (Sec. IV) in order to ascertain the degree to which the captured shock wave structure corresponds to that given by the analytical solutions. Toward that end, the composite analytical solution, valid to $\mathcal{O}\left(\epsilon^{2}\right)$, formulated in the previous section may be used to generate solutions at various Reynolds numbers. The value of $c_{2 u}$ is taken as $m u_{1 x}(0)$ so that $u$ remains finite at the channel throat. The value of $c_{2 d}$ is given by Eq. (44) with the sonic line positioned at $x_{p 0}=0.8$. In the analytical solutions, the Prandtl number, as well as the exponent $k_{v}$ in the viscosity relation, is taken as unity.

Figure 3 is a comparison of the axial velocity along the centerline as determined from the theory for flows at moderate Reynolds number with the numerical solution. Analytical solutions for three values of the parameter $m$, which is inversely proportional to Reynolds number, are shown, namely, $m=0.05,0.1$, and 0.2 , corresponding to Reynolds numbers of 389,194 , and 97 , respectively. The numerical solution shown is obtained using an artificial viscosity coefficient $\alpha$ of 0.5 . The analytical solutions are seen to bracket the numerical solution within the shock structure region; the numerical solution also passes through sonic velocity at the location of the shock wave in inviscid flow. Additionally, the overall shape of the curves found analytically and numerically are qualitatively similar,

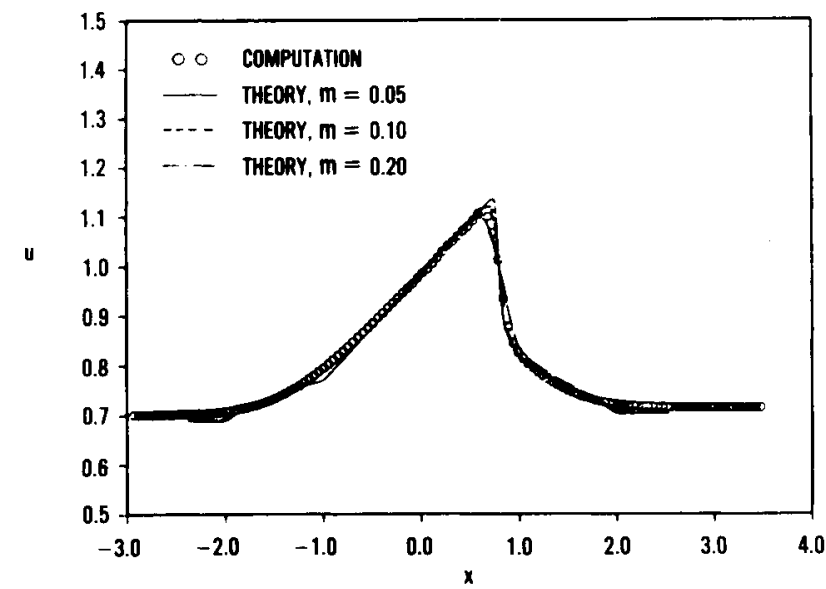

Fig. 3 Centerline axial velocity comparison between composite viscous flow theory and numerical solution.

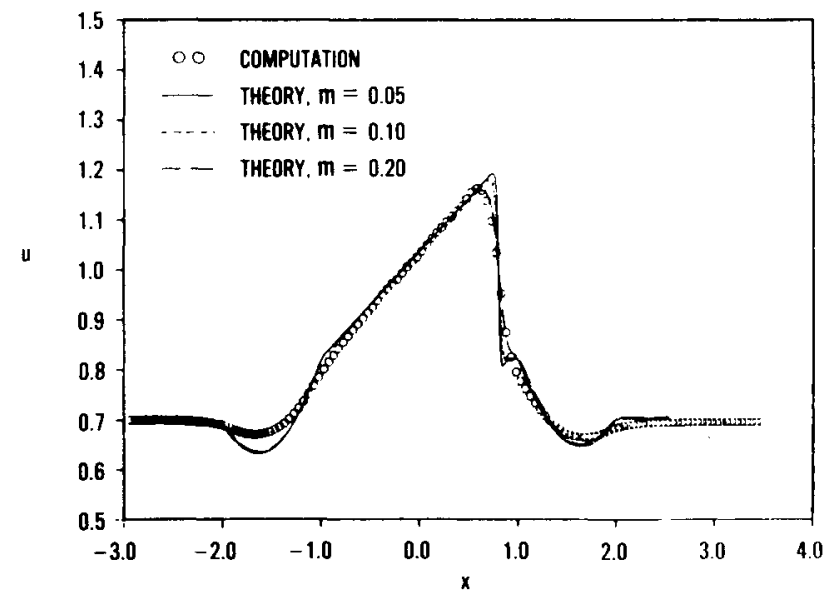

Fig. 4 Wall axial velocity comparison between composite viscous flow theory and numerical solution. especially within the shock wave structure. A comparison between the analytical and numerical solutions for the axial velocity component along the wall is found in Fig. 4. Again, the overall character of the numerical solution is quite similar to that displayed by the analytical solutions. In this particular example, the numerical solution does not exhibit the Zierep singularity, ${ }^{6}$ probably as a result of excessive numerical damping. In light of these favorable comparisons, it is evident that a numerical solution of the Euler equations, including artificial viscosity and truncation error, for a transonic channel flow containing a shock wave is similar to the analytical solution of the Navier-Stokes equations at moderate Reynolds numbers.

The effects on the numerical solution of changes in the magnitude of the artificial viscosity is shown in Fig. 5. Here the axial velocity component $u$ on the centerline is shown for three values of the artificial viscosity coefficient $\alpha$ and compared with the analytical solution for inviscid flow in the channel (Sec. III). As the numerical damping increases, the numerical shock wave is seen to increase in thickness in the expected manner. Except for the largest damping solution $(\alpha=1)$, the sonic points within the numerical shock wave structures are observed to coincide with the location of the discontinuous shock wave in the inviscid flow solution. The numerical shock wave obtained with an artificial viscosity coefficient of 1.0 is approximately $3 \epsilon$ in thickness; it may be an indication of the limit to which the amount of artificial viscosity may be increased and still give results similar to those obtained in the physical flow as Reynolds number decreases.

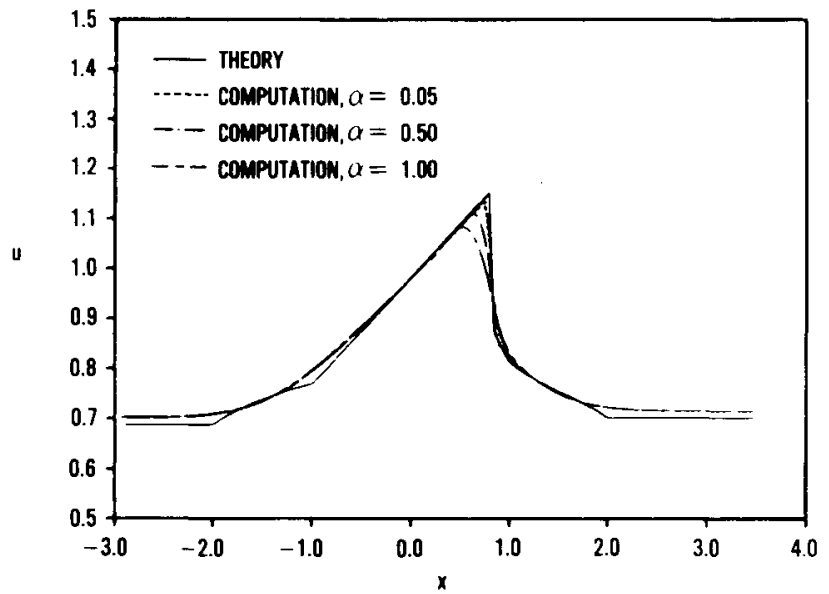

Fig. 5 Centerline axial velocity comparison between composite viscous flow theory and numerical solutions.

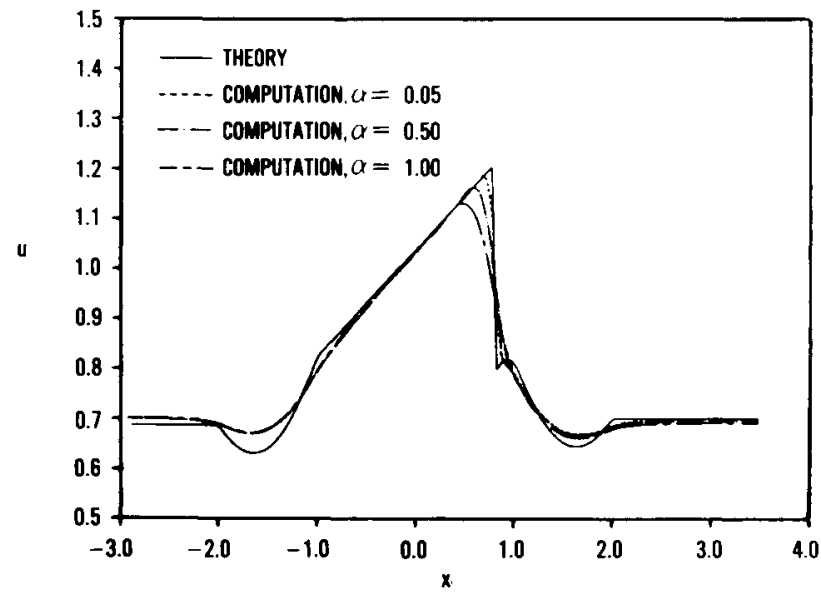

Fig. 6 Wall axial velocity comparison between composite inviscid flow theory and numerical solutions. 
The effects of numerical damping on the axial velocity at the wall are found in Fig. 6. Here, the presence of the Zierep singularity in the numerical solution is observed for the case with the smallest damping ( $\alpha=0.05)$. Again, the sonic points within the numerical shock structures coincide with the location of the shock wave discontinuity except for the solution with high damping.

\section{Conclusions}

The behavior of shock waves in transonic flows with varying Reynolds and Prandtl numbers has been examined using analytical and numerical methods. The method of matched asymptotic expansions has been used to provide analytical solutions for both an inviscid and a viscous, thermally conducting transonic flow in a symmetric channel. The extension of previous work on viscous transonic flows in a channel to include the effects of Reynolds and Prandtl numbers upon the structure of a shock wave at moderate Reynolds number has been presented. The position of the sonic line within the structure of a shock wave has been found to first order and is observed to be independent of Reynolds and Prandtl numbers to this order. Further, one finds that it is coincident with the position of the shock wave in the corresponding inviscid flow.

Numerical solutions for an inviscid, transonic flow in a channel have been obtained using a second-order accurate, finite volume method. The numerical solutions have been observed to agree well with the composite analytical solutions for the channel flow including the structure of the shock wave. Additionally, the position of the sonic line within the numerical shock structure agrees well with the position of the shock wave in inviscid flow as determined analytically. Although not presented here, similar results were found to zeroth order in the problem of transonic flow about an airfoil with a supercritical region terminated by a weak shock wave. ${ }^{7}$

This research lends support to the commonly accepted supposition that the position of the sonic line within the structure of a numerical shock wave can be adopted as the location of the corresponding shock wave in inviscid flow. Finally, the analytical solutions presented here allow this demanding model problem to be used in validating numerical algorithms.

\section{Acknowledgments}

The authors wish to thank Prof. Arthur F. Messiter of the University of Michigan and Drs. Wilbur Hankey and Donald Rizzetta of the Flight Dynamics Laboratory for their stimulating discussions and suggestions during the course of this work.

\section{References}

${ }^{1}$ Warming, R. F. and Hyett, B. J., "The Modified Equation Approach to the Stability and Accuracy Analysis of Finite-Difference
Methods," Journal of Computational Physics, Vol. 14, 1974, pp. 159-179.

${ }^{2}$ Illingworth, C. R., "Shock Waves," Modern Developments in Fluid Mechanics High Speed Flow, Vol. I, edited by L. Howarth, Oxford Press, Oxford, England, 1953, pp. 105-137.

${ }^{3}$ Holst, T. L., "Fast, Conservative Algorithm for Solving the Transonic Full-Potential Equation," AIAA Journal, Vol. 18, Dec. 1980, pp. 1431-1439.

${ }^{4}$ Holst, T. L. and Ballhaus, W. F., "Fast, Conservative Schemes for the Full Potential Equation Applied to Transonic Flows," $A I A A$ Journal, Vol. 17, Feb. 1979, pp. 145-152.

${ }^{5}$ Adamson, T. C. Jr., "A Study of Unsteady Transonic Flows with Shock Waves in Two Dimensional Channels," University of Michigan, Ann Arbor, Tech. Rept. MICH-10-PU, Final Technical Report for Office of Naval Research, Contract N0004-67A-0226-0005, Nov. 1972.

${ }^{6}$ Messiter, A. F. and Adamson, T. G. Jr., "On the Flow Near a Weak Shock Wave Downstream of a Nozzle Throat," Journal of Fluid Mechanics, Vol. 69, 1975, pp. 97-108.

${ }^{7}$ Mace, J. L., "Shock Waves in Transonic Flows at Moderate Reynolds Number," Ph.D. Thesis, University of Michigan, Ann Arbor, 1984.

${ }^{8}$ Taylor, G. I., "The Conditions Necessary for Discontinuous Motion of Gases," Proceedings of the Royal Society of London, Ser. A, Vol. 84, 1910, pp. 371-377.

${ }^{9}$ Szaniawski, A., "The Structure of a Weak Shock-Wave in a Viscous Heat Conducting Fluid," Archives of Mechanics, Vol. 18, No. 2, 1966, pp. 127-144.

${ }^{10}$ Sichel, M., "Two-Dimensional Shock Structure in Transonic and Hypersonic Flow," Advances in Applied Mechanics, 11th ed., edited by C. S. Yih, Academic Press, New York, 1971, pp. 131-207.

${ }^{11}$ Sichel, M., "The Effect of Longitudinal Viscosity on the Flow at a Nozzle Throat," Journal of Fluid Mechanics, Vol. 25, 1966, pp. 769-786.

${ }^{12}$ Adamson, T. C. Jr. and Messiter, A. F., "A Study of Hybrid Computing Techniques for Transonic Flow Fields," University of Michigan, Ann Arbor, Rept. UM 016294-F, Final Technical Report prepared for the Air Force Office of Scientific Research, Grant 78-3635, Feb. 1980.

${ }^{13}$ Vincenti, W. G. and Kruger, C. H. Jr., Introduction to Physical Gas Dynamics, John Wiley \& Sons, New York, 1965, Chap. 10.

${ }^{14}$ Schlichting, H., Boundary-Layer Theory, McGraw-Hill Book Co., New York, 1968, Chap. 13.

${ }^{15}$ Wylie, C. R. Jr., Advanced Engineering Mathematics, McGrawHill Book Co., New York, 1966, Chap. 12.

${ }^{16}$ Visbal, M. and Knight, D., "Generation of Orthogonal and Nearly Orthogonal Coordinates with Grid Control Near Boundaries," AIAA Journal, Vol. 20, March 1982, pp. 305-306.

${ }^{17}$ MacCormack, R. W. and Paullay, A. J., "Computational Efficiency Achieved by Time Splitting of Finite Difference Operators," AIAA Paper 72-154, Jan. 1972.

${ }^{18}$ MacCormack, R. W. and Baldwin, B. A., "A Numerical Method for Solving the Navier-Stokes Equations with Application to Shock-Boundary Layer Interactions," AIAA Paper 75-1, Jan. 1975.

${ }^{19}$ Liepmann, H. W. and Roshko, A., Elements of Gasdynamics, John Wiley \& Sons, New York, 1967, Chap. 7.

${ }^{20}$ Rudinger, G., Nonsteady Duct Flow, Dover Publications, New York, 1969, Chap. 6. 